\title{
ESPACES PEINTS, PÈLERINAGE ET LITURGIE À LA CATHÉDRALE NOTRE-DAME DU PUY (XI ${ }^{\mathrm{e}}-\mathrm{XIII}^{\mathrm{e}}$ SIÈCLES $)^{1}$
}

\section{MARIE CHARBONNEL}

UDC: $726.6(445 \cdot 93) " 10 / 12 "$ 75.052

Original scientific paper

Manuscript received: 10. 02. 2017.

Revised manuscript accepted: 20. 02. 2017.

DOI: 10.1484/J.HAM.5.113764
M. Charbonnel

38 rue Blaise Pascal

63000 Clermont-Ferrand, France marie.charbonnel@icloud.com

The cathedral at Le Puy is an emblematic monument of the western Middle Ages. The aim of this paper is a reinterpretation of its wall-paintings as spatial markers of a topography surrounded by an ecclesiological and liturgical discourse, translated by images and inscriptions, and deeply linked to the cathedral's various statuses, as a cathedral and as a pilgrimage place.

Keywords : Le Puy-en-Velay, cathedral, wall-painting, liturgy, pilgrimage, epigraphy.

La double fonction de Notre-Dame du Puy, qui est à la fois édifice de pèlerinage et cathédrale, induit une belle diversité d'enjeux, à laquelle s'ajoute la bonne conservation de ses vestiges de peintures murales. L'édifice constitue de facto un terrain d'investigation privilégié pour l'étude des liens qui unissent le décor monumental et les pratiques mise en œuvre au sein de l'espace sacré médiéval. En effet, les peintures sont le reflet, à travers les thèmes iconographiques et épigraphiques choisis, de ce que les commanditaires ont souhaité montrer de la cathédrale et des manifestations liturgiques qui s'y déroulaient. Le pèlerinage à la Vierge a façonné en grande partie le groupe cathédral que nous connaissons aujourd'hui. Bernard, écolâtre d'Angers, livre son regard sur la ville vers 1020 : « célèbre et populeuse ville dont l'ancien nom, presque oublié, est, si je ne me trompe, Anicium et qu'on appelle vulgairement le Puy de Sainte-Marie $»^{2}$. La liturgie cathédrale, le culte rendu à la Vierge, l'inscription du site dans un réseau de pèlerinage ainsi que son rayonnement, ont conditionné l'élaboration des peintures murales qui se répartissent dans divers espaces et durant plusieurs siècles, au gré des aménagements et réaménagements dans la cathédrale. En fonction des zones considérées, les peintures constituent le décor originel, premier épiderme de l'édifice, ou résultent de réfections. Nous reviendrons sur ces différentes phases (fig. 1), en débutant à l'ouest par le porche et les chapelles Saint-Gilles et Saint-Martin, pour allervers l'est, le transept et ses tribunes ; ce déplacement est l'occasion de dessiner une topographie discursive, mnémonique et apo- tropaïque de l'édifice en prenant en compte la fonction de chacun des pôles. Le rôle joué par la peinture murale dans l'établissement d'un dialogue entre intérieur et extérieur, à travers l'espace de passage que constitue le porche, ainsi que les fonctions paraliturgiques de cet espace doivent d'abord être interrogés. Les phénomènes de résonnance liant les images et inscriptions du décor et les gestes et paroles de la liturgie seront ensuite abordés à travers les décors intérieurs de la cathédrale³.

\section{LES PEINTURES DES PARTIES OCCIDENTALES ET LE RAYONNEMENT DE LA CATHÉDRALE}

La première peinture sous le porche occidental est la représentation de saint Christophe (fig. 2), datable de la fin du XIII ${ }^{\mathrm{e}}$ siècle, située au niveau de la deuxième travée du collatéral du porche, à l'entrée de la chapelle Saint-Gilles. Cette peinture est composée d'une image du saint, très largement lacunaire en raison de la destruction partielle du mur qui l'accueille, et d'une formule expiatoire, peinte au sommet de la pile située à l'entrée de la chapelle Saint-Gilles : Christofori sanctam speciem quicumque tuetur illo nempe die nullo langore tenetur ${ }^{4}$. L'association de saint Christophe à des espaces extérieurs est fréquente ; l'image est parfois située sur des zones de passage périlleuses comme dans les montagnes alpines ${ }^{5}$. Ce thème, par sa valeur apotropaïque, a donc toute sa place dans un édifice fréquenté par des pèlerins qui ont entrepris un voyage difficile.

\footnotetext{
${ }^{1}$ Qu'il nous soit permis de remercier ici François Heber-Suffrin, Vincent Debiais et Pascale Chevalier pour leurs relectures attentives.

${ }^{2}$ M. DURLIAT, La cathédrale du Puy, in Congrès archéologique de France, 133 session, 1975, Velay, Paris, 1976, p. 55-163 ; A. BOUILLET (éd.), Liber miraculorum sancte Fidis, Paris, 1897, I, 6.

3 Nous signalons au lecteur que lorsqu'une restitution complète des inscriptions a été possible, l'édition critique est présentée en note. Les lacunes sont restituées entre crochets, les abréviations développées entre parenthèses et les éventuelles corrections apportées entre accolades, selon les normes de l'édition critique épigraphique présentées par Cécile Treffort en introduction du volume 23 du Corpus des inscriptions de la France médiévale. Côtes-d'Armor, Finistère, Ille-et-Vilaine, Morbihan, Loire-Atlantique et Vendée, Paris, 2008.

4 [Christ] ofori s(anc)ta(m) speciem q(u)icu(m)que tue(tur) illo ne[mpe die] nullo la[ng]ore tenetu[ $r$ ]

${ }_{5}^{5}$ D. RIGAUX, Une image pour la route. L’íconographie de saint Christophe dans les régions alpines (XIIe-XVe siècle), in Voyages et voyageurs au Moyen Age, Actes du $26^{e}$ congrès de la Société des historiens médiévistes de l’enseignement supérieur public (Aubazine, 1996), Paris, 1996, p. 235 -266.
} 


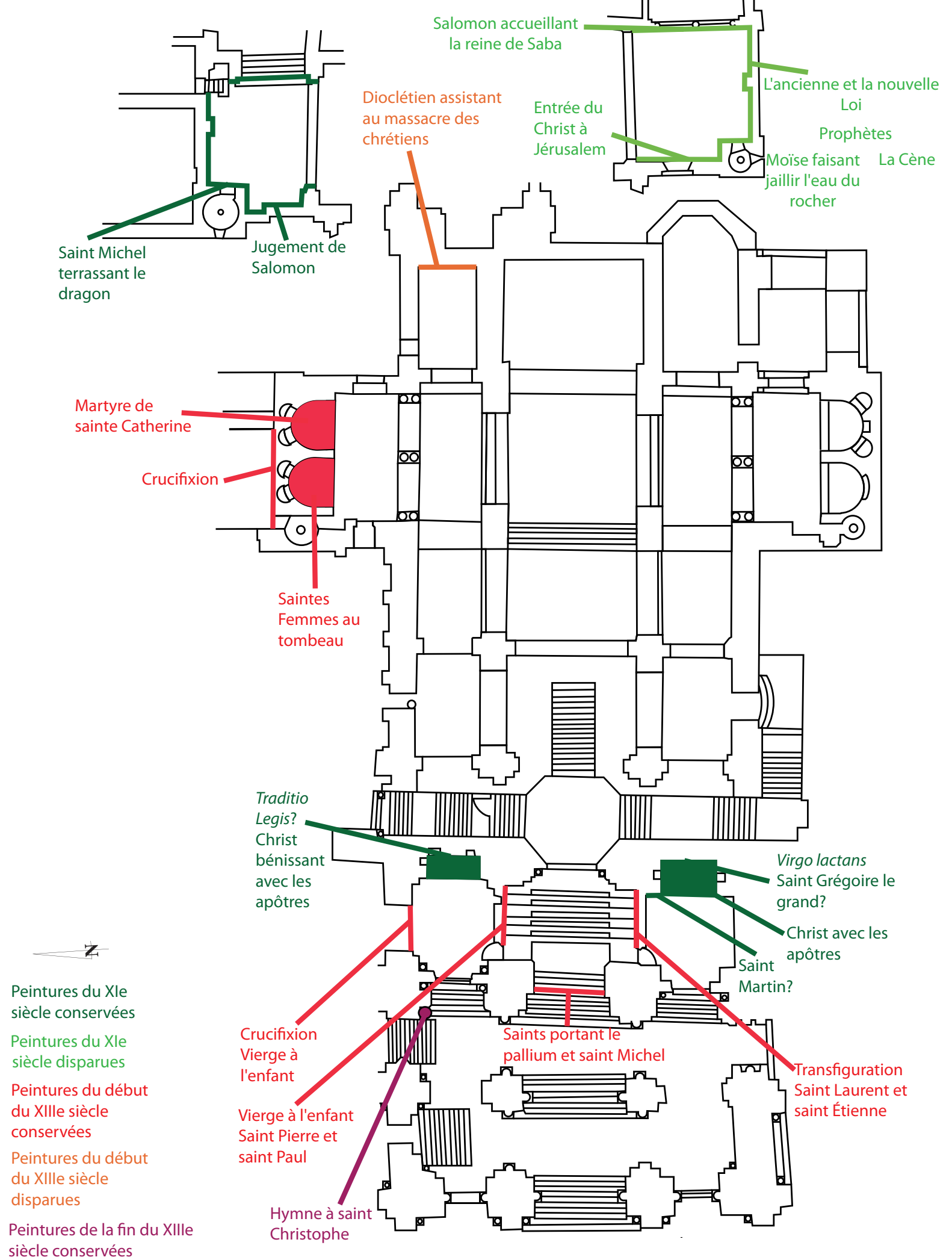

Fig. 1 - Plan de la cathédrale du Puy, situant les thèmes iconographiques des peintures murales (del. M. Charbonnel)

Les deux chapelles Saint-Gilles et Saint-Martin contiennent elles aussi des peintures qui, en raison de leur caractère lacunaire, n'ont pas beaucoup retenu l'attention, mais qu'il convient d'analyser. Le choix de la titulature de la chapelle Saint-Gilles pourrait être le résultat d'une demande de Raymond de Saint-Gilles, comte de Toulouse. Celui-ci, sur le point de partir pour la croisade en 1096, fait un don important à la cathédrale ; il demande que la fête de saint Gilles y soit célébrée chaque année et qu'une lampe y soit disposée ante Dei Genitricis venerandam imaginem super altare $^{6}$. La chapelle a connu plusieurs phases de décor peint. La première, datable de la seconde moitié du $\mathrm{XI}^{\mathrm{e}}$ siècle, pourrait avoir suivi de près la donation du comte de Toulouse, sans que soit mentionnée son édification. Seules

${ }^{6}$ I. H. FORSYTH, Throne of Wisdom. Wood sculptures of the Madonna in Romanesque France, Princeton, 1972, p. 104. 


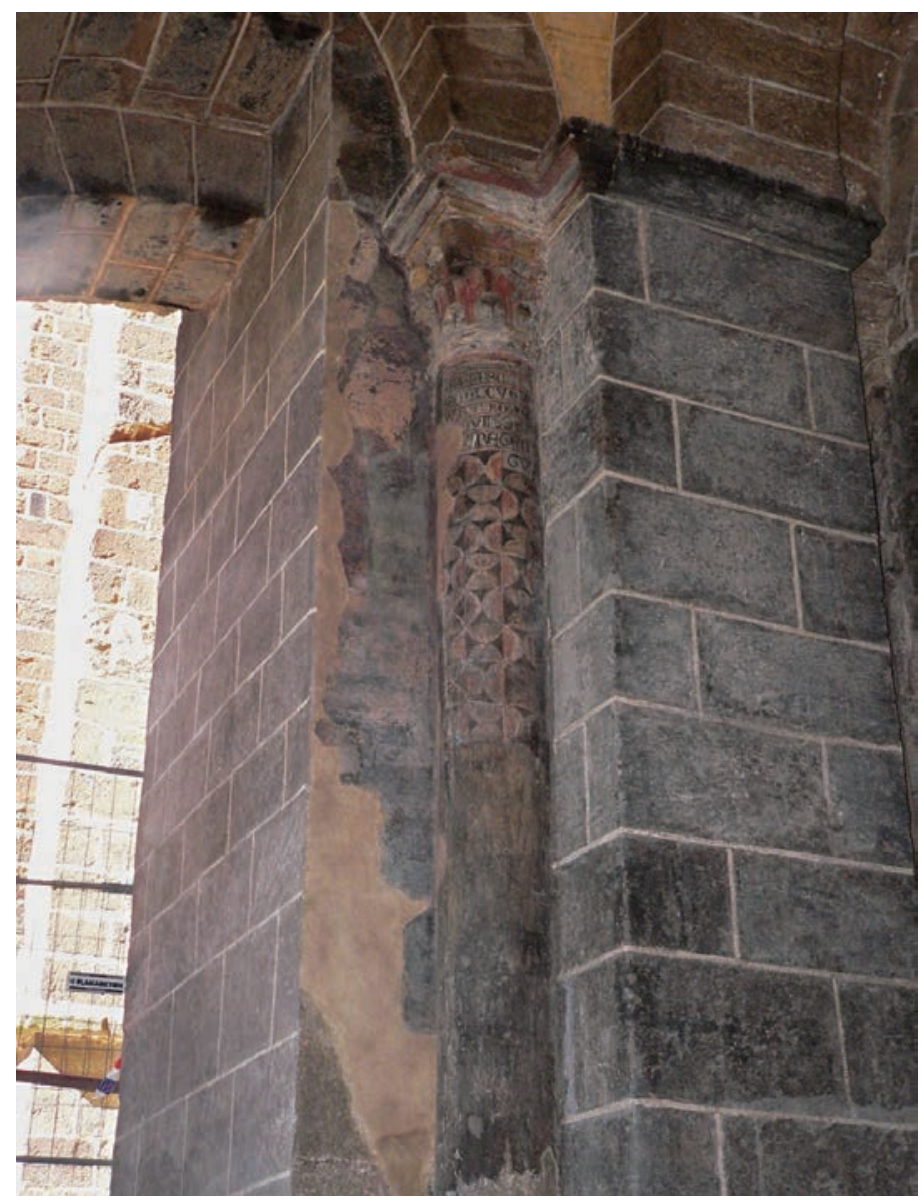

Fig. 2 - Porche de la cathédrale, hymne à saint Christophe (cl. M. Charbonnel)

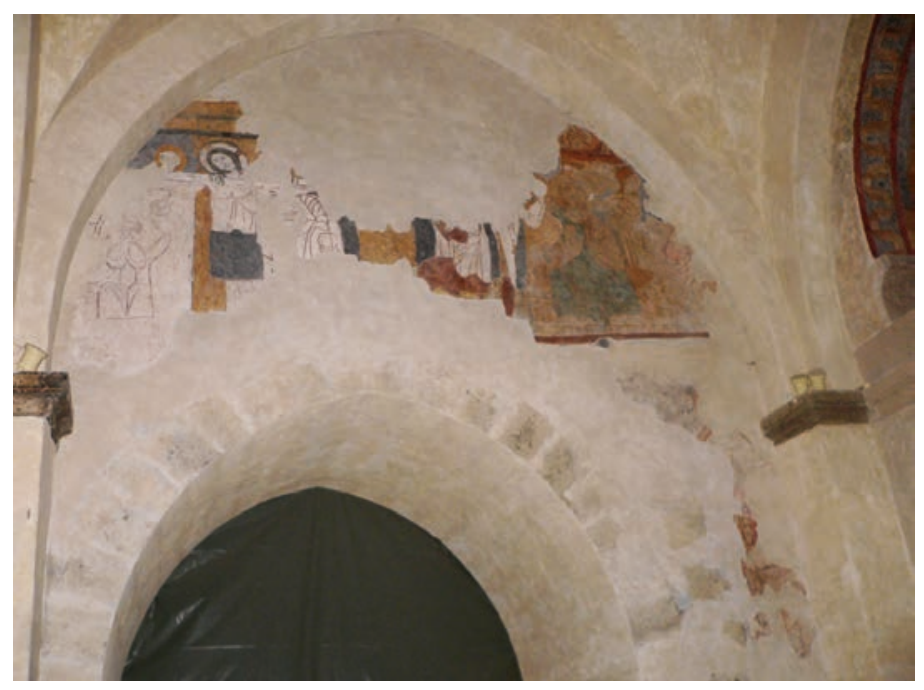

Fig. 4-Chapelle Saint-Gilles, Crucifixion (cl. M. Charbonnel)

quelques bribes de cette phase demeurent aujourd'hui, sur le mur nord, à l'est de la porte donnant accès au bâtiment des Mâchicoulis, dont le percement, dans le courant du XIII ${ }^{\mathrm{e}}$ siècle, a perturbé les peintures. L'inscription [S]tefato (fig. 3) est le seul élément permettant d'identifier sur ce mur une Crucifixion pour la première phase. Le thème de la Crucifixion (fig. 4) a été réinstallé après la destruction des premières peintures et déplacé au-dessus de la porte de communication, témoignant de la volonté de pérenniser certaines associations entre espaces et iconographie. D’autres

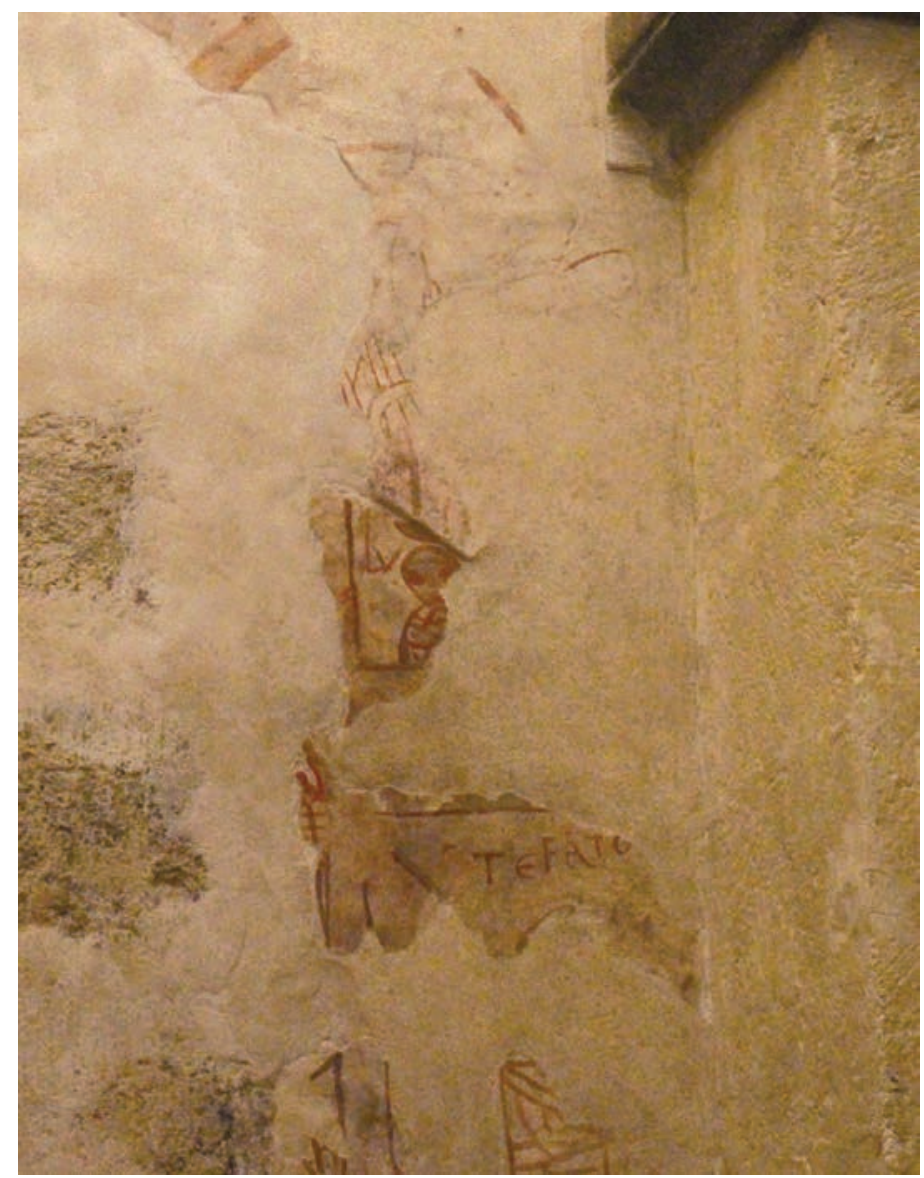

Fig. 3 - Chapelle Saint-Gilles, Stéfaton (cl. M. Charbonnel)

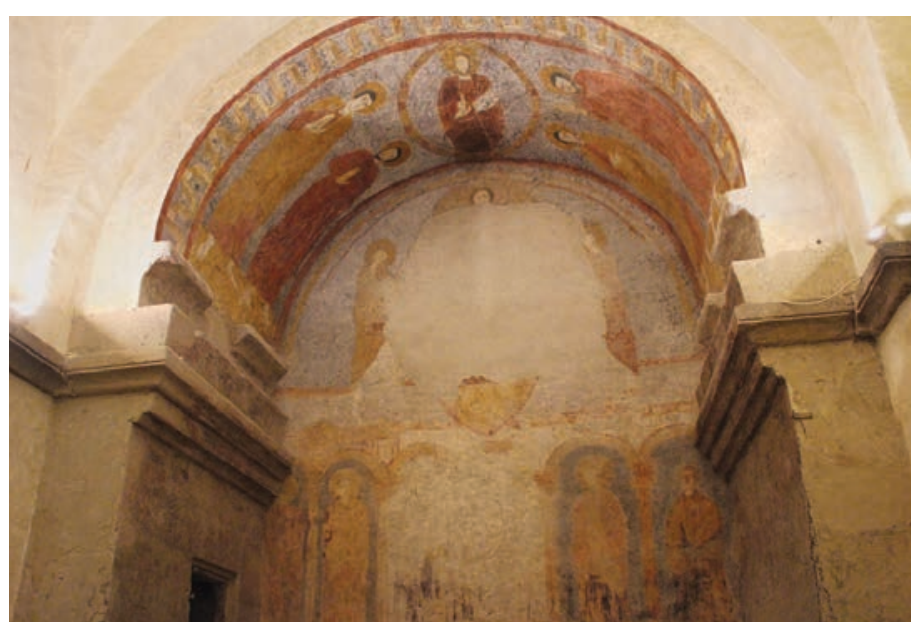

Fig. 5 - Chapelle Saint-Gilles, traditio legis avec les apôtres? (cl. M. Charbonnel)

peintures, appartenant également à la seconde campagne, occupent le mur oriental. L'ensemble est très lacunaire. Le Christ apparaît dans une mandorle, encadré par deux personnages (fig. 5). L'intrados de l'arc est occupé par un Christ bénissant entouré des apôtres, disposés de la manière suivante : quatre sur l'intrados et six sur le mur. Les deux personnages de part et d'autre du Christ viendraient alors compléter le collège. Ces éléments pourraient constituer une Traditio Legis, le don de la Loi à saint Pierre par le Christ. Ce thème a souvent été utilisé pour valoriser la figure du pape, en rappelant son statut de successeur de saint Pierre et en renforçant ainsi sa légitimité7. Au Puy, il illustre probable-

7 Voir notamment les travaux d'H. TOUBERT, Les fresques de la Trinité de Vendôme, un témoignage sur l'art de la réforme grégorienne, in Cahiers de Civilisation Médiévale, 26-104, 1983, p. 297-326. 


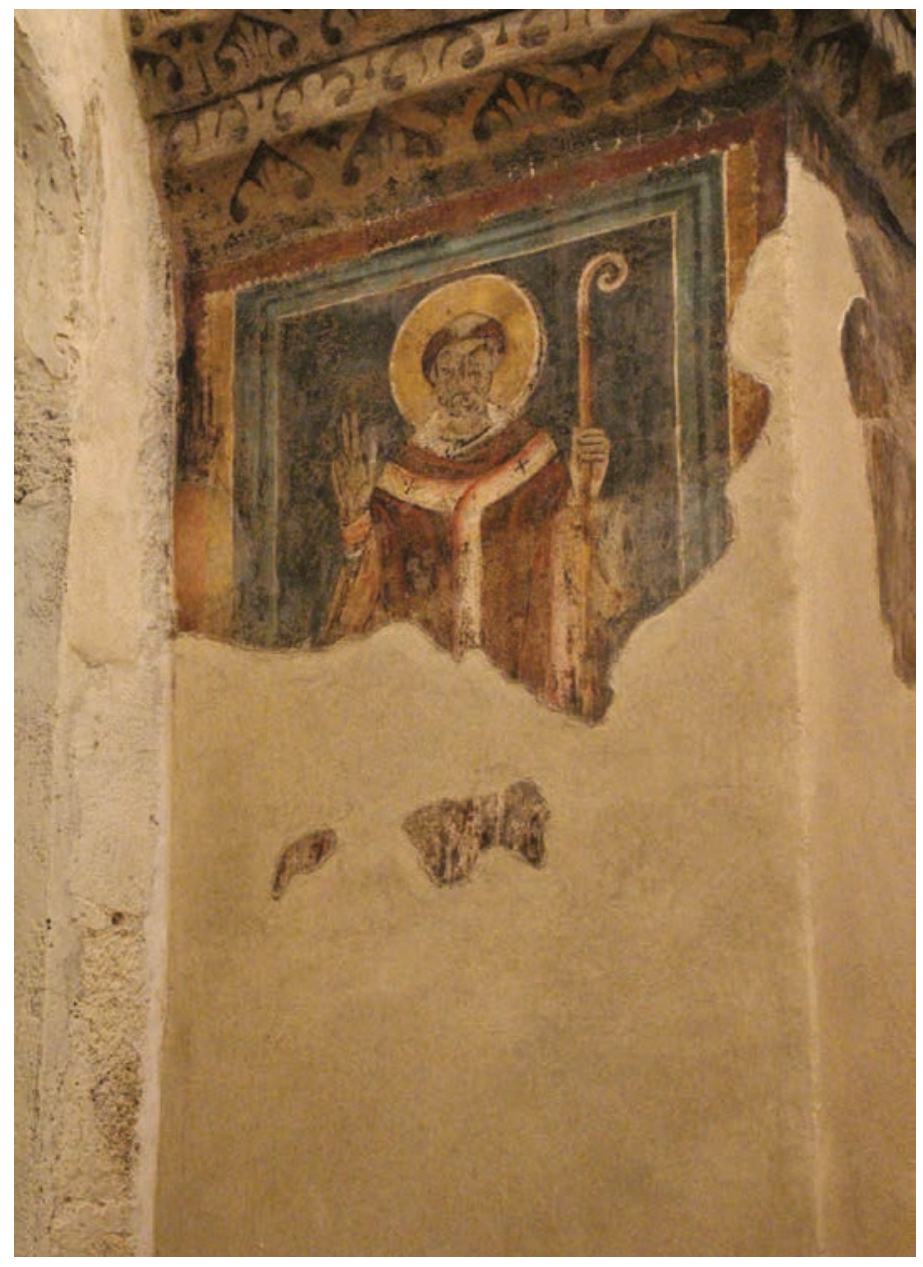

Fig. 6-Chapelle Saint-Martin, saint Martin? (cl. M. Charbonnel)

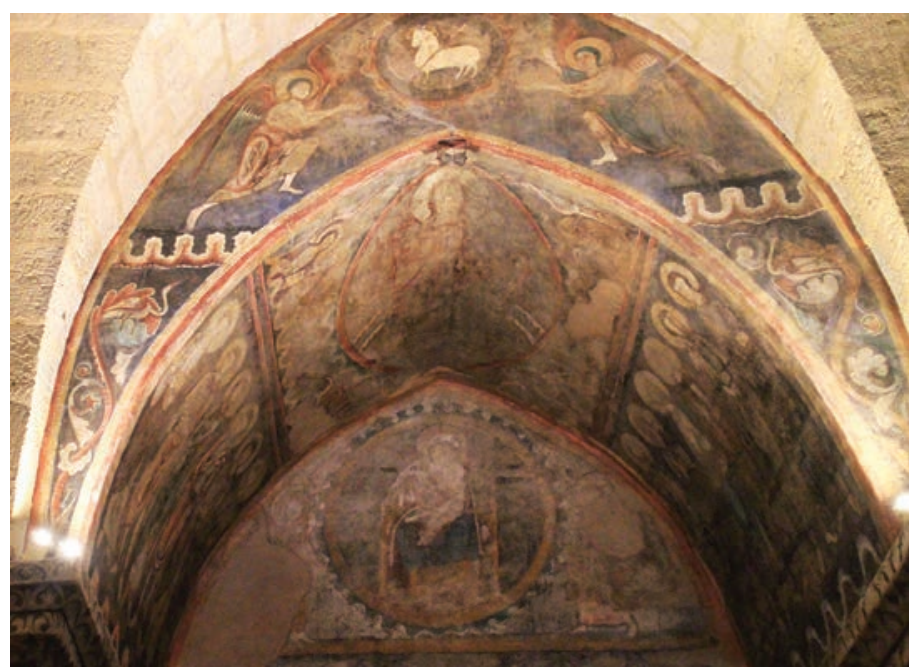

Fig. 8 - Chapelle Saint-Martin, le Christ et les apôtres (cl. M. Charbonnel)

ment les relations privilégiées entretenues avec la papauté, relations qui se traduisent par le rattachement direct du diocèse au Saint-Siège par Sylvestre II en 999, l'attribution du pallium à l'évêque du Puy par Léon IX en 1051, ou encore la nomination d'Adhémar de Monteil, évêque du Puy, par Urbain II comme légat pontifical pour promouvoir la première croisade vers 1095, et la visite de Calixte II en 1119. Ainsi, cette

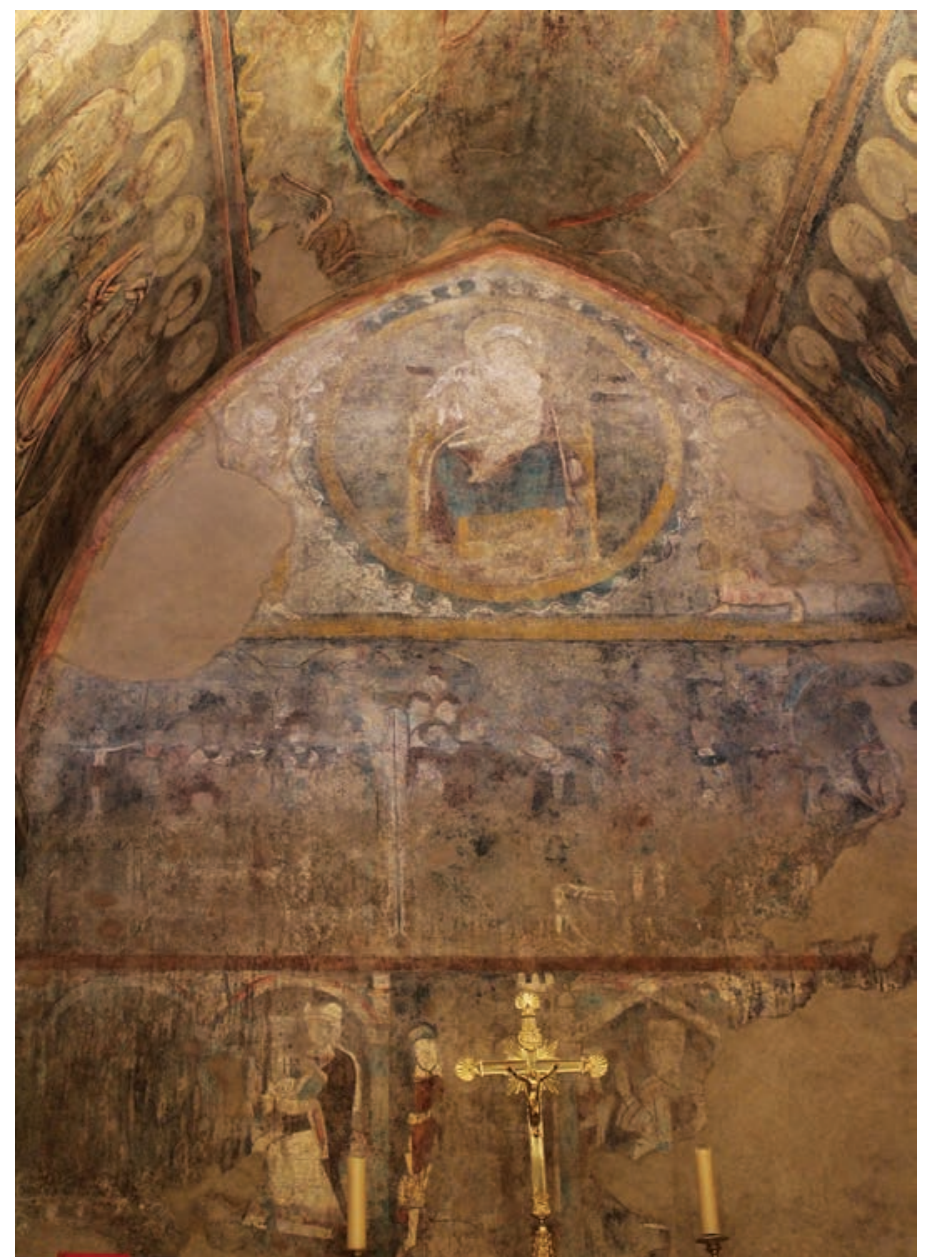

Fig. 7 - Chapelle Saint-Martin, la virgo lactans, Grégoire le grand? (cl. M. Charbonnel)

peinture vient valoriser le rattachement direct de l'évêché à la papauté ${ }^{8}$. Les bonnes relations du Puy avec Rome sont d'ailleurs étayées par les propos de Léon IX, proches dans le temps de l'élaboration des peintures : « Nulle part, dans aucun autre des sanctuaires qui lui sont dédiés, Marie ne reçoit un culte plus spécial et plus filial de respect, d'amour et de vénération que celui qui lui est rendu par les fidèles de la Gaule entière dans cette église du Mont Anis, dénommée encore du Velay ou du Puy-Sainte-Marie $» 9$.

Les conditions de la fondation de la chapelle Saint-Martin sont, quant à elles, plus difficiles à cerner. Un saint est représenté au nord de l'arc aveugle, il s'agit peut-être du titulaire de la chapelle (fig. 6). Le mur oriental présente une Virgo lactans, inscrite sous un profond arc aveugle ; au-dessous, les scènes sont difficilement identifiables (fig. 7). Dans la première scène, une assemblée se tient devant le Crucifix. Leur disposition et les rares fragments lisibles inviteraient à les associer à un cycle hagiographique, peut-être celui de saint Grégoire le Grand. L'intrados de l'arc est occupé par un Christ en majesté accompagné des apôtres, tandis que l'Agneau s'élève dans une mandorle tenue par deux anges au niveau de l'archivolte (fig. 8) ; on y devine encore une inscription très lacunaire, lisible sur le relevé effectué en 1953 par Marcel Nicaud ${ }^{10}$. Cette association de motifs ico-

\footnotetext{
${ }^{8}$ D. RUSSO, Espace peint, espace symbolique, construction ecclésiologique. Les peintures de Berzé-la-Ville (Chapelle-des-Moines), in Revue Mabillon, 11-72, 2000, p. 57-87.

${ }_{9}$ Gallia Christiana, II, col. 228.

1o ---]omnia[---
} 
nographiques pourrait être une allusion à la double nature du Christ, à l'instar des peintures du porche.

Des peintures du début du XIII ${ }^{\text {e }}$ siècle sont présentes sur l'intrados de la troisième travée du porche (fig. 9). Leur iconographie associe saint Michel dans un médaillon à deux saints portant le pallium qui l'encadrent. Sous la quatrième travée du porche, deux thèmes se font ensuite face : la Vierge en majesté au nord (fig. 1o) et la Transfiguration sur le mont Thabor au sud (fig. 11). Les inscriptions qui accompagnaient la Vierge en majesté font défaut aujourd'hui, mais la présence d'Isaïe et de saint Jean-Baptiste laisse toutefois présager de leur contenu. Comme l'ont suggéré Paul Deschamps et Marc Thibout, les textes d'Isaie et Jean-Baptiste sont probablement proches des formulations utilisées à Conques, pour les mêmes personnages, dans le cadre d'une Annonciation, à savoir, Dixit Isaias : exiet virga de radica Jesse et Johannes ait : ecce Agnus Dei ${ }^{11}$. Des clichés anciens laissent d'ailleurs apparaître un Virg[-] sur le phylactère d'Isaïe. L'intrados de l'arc est, quant à lui, occupé par un Christ bénissant entre deux anges, et par saint Pierre et saint Paul.

Le texte associé à la Transfiguration est mieux conservé. En plus de la traditionnelle parole de Pierre, Domine bonum est nos hic esse ${ }^{12}$, deux anges déploient des phylactères au dessus de la scène. L'ange de gauche porte aujourd'hui la formule Vox pa[...] De[...] sonat ai[-] p(ro)phe[..], mais la comparaison avec des clichés anciens montre que la restauration a sans doute altéré l'inscription ; voilà pourquoi nous reprenons la lecture de Marc Thibout et Paul Deschamps : Vox Isaias hoc est verum sonat animo prophete ${ }^{13}$. Quant à l'ange de droite, il porte la formule suivante [---] tra(n) sformatur vero vo(b)is esse $p$ (ro)batur. Sur l'arc au dessus de la scène se trouvent les diacres, continuateurs de l'œuvre du Christ : saint Laurent et saint Étienne. L'utilisation de cette iconographie peut avoir pour but d'insister sur l'exceptionnelle maternité de la Vierge, en employant un thème où la nature divine du Christ se manifeste durant son existence terrestre, tout en accentuant les relations entre Ancien et Nouveau Testaments (dans la représentation de la Vierge accompagnée d'Isaïe et de saint Jean-Baptiste) et la continuité de l'œuvre du Christ avec les diacres Étienne et Laurent. Les deux thèmes de la Vierge à l'Enfant et de la Transfiguration se répondent et mettent en scène - à l'instar des portes des chapelles Saint-Gilles au nord et Saint-Martin au sud, situées dans les deux travées précédentes et respectivement consacrées à l'Enfance du Christ et à la Passion - l'histoire du Christ, de l'Incarnation au nord, à la Rédemption au sud ${ }^{14}$. Le point de vue liturgique est éclairé par le Prosolarium ecclesiae Aniciensis, manuscrit du $\mathrm{XVI}^{\mathrm{e}}$ siècle détaillant l'office de la Circoncision,

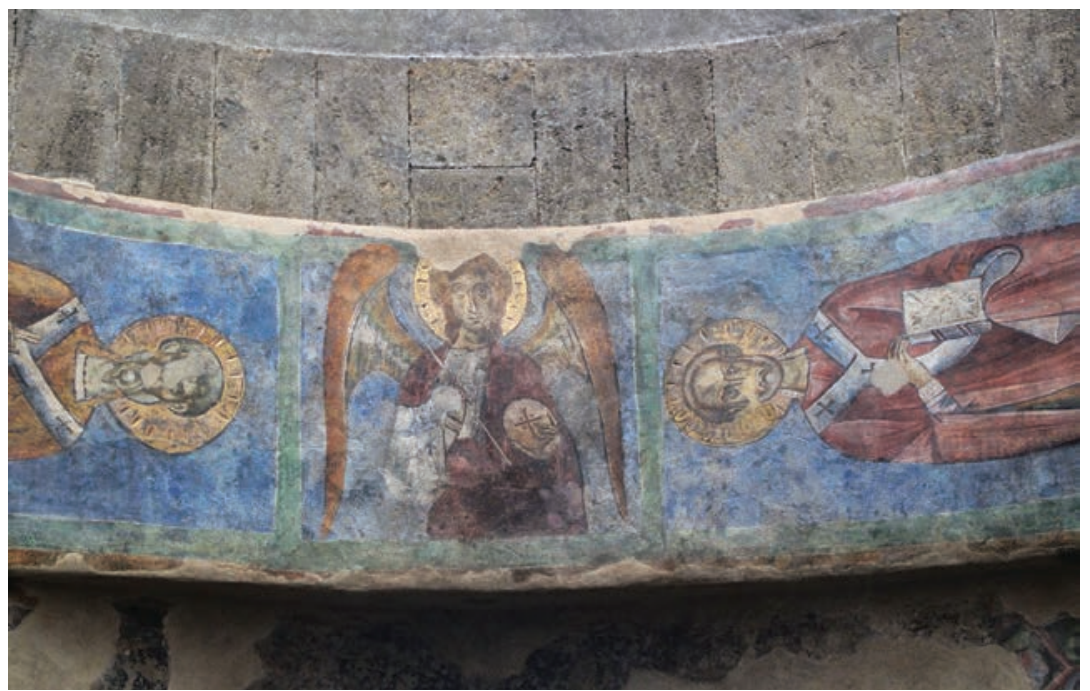

Fig. 9 - Porche, saint Michel et deux diacres (cl. M. Charbonnel)

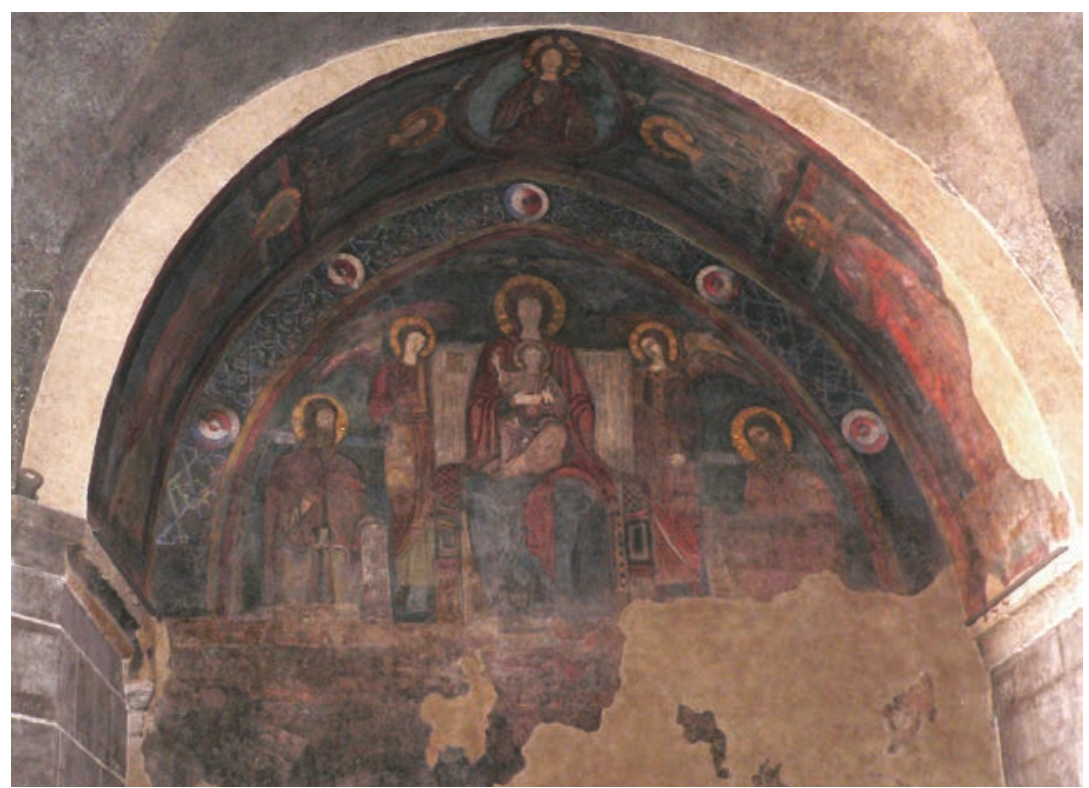

Fig. 10 - Porche, la Vierge en majesté (cl. M. Charbonnel)

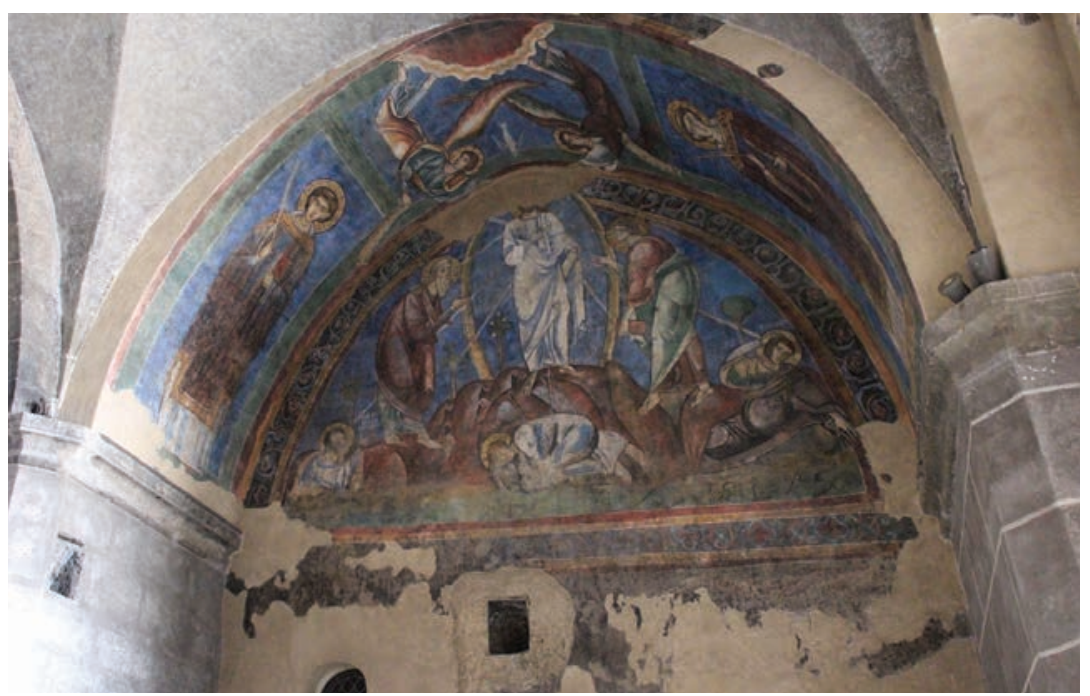

Fig. 11 - Porche, la Transfiguration (cl. M. Charbonnel)

"P. DESCHAMPS, M. THIBOUT, La peinture murale en France au début de l'époque gothique, Paris, 1963, note 1 p. 83.

${ }^{12} \mathrm{D}$ (omi)ne bonu(m) e(st) nos [hic] esse.

${ }^{13}$ Ibidem, p. 84 ; Vox Isaias $[h]$ oc e $[s]$ t verum sonat a $[n] i[m]$ o prophete.

${ }^{14}$ X. BARRAL I ALTET, La cathédrale du Puy-en-Velay, Genève-Paris, 200o, p. 266 ; H. SCHRADE, La peinture romane, Bruxelles, 1966, p. 150-151. 
probablement composé au XII siècle pour la cathédrale du Puy selon Wulf Arlt. L'office se déroule à la cathédrale durant vingt-quatre heures entre le 31 décembre et le $1^{\mathrm{er}}$ janvier. Le manuscrit mentionne au folio 96r l'usage de l'espace devant ces peintures pour des stations. Ainsi, à prime, l'antienne Salve Regina est récitée in gradibus coram beate Marie, puis c'est le tour de l'antienne Domine bonum est nos hic esse. Si vis faciamus hic tria tabernacula, tibi unum, Moyse unum et Helye unum, in gradibus coram Transfigurationem $D_{\text {Domini }}{ }^{15}$. Le manuscrit fait état de la station suivant immédiatement celle-ci, coram ymagine beate Marie, sans plus de précision, genou fléchi, en récitant une oraison. Il est particulièrement intéressant de noter que la présence de cette antienne dans l'office de la Circoncision du Christ est un unicum. L'inscription du XIII ${ }^{\mathrm{e}}$ siècle a pu ici influer sur la composition de l'office au siècle suivant.

Au-delà de la lecture d'ensemble liant Incarnation et Rédemption et des aspects liturgiques a posteriori, le choix du thème de la Transfiguration peut être lié à d'autres enjeux. En effet, le Codex Calixtinus, largement diffusé en Europe au moment de l'exécution des peintures, a fortiori au Puy puisque le pèlerinage effectué par l'évêque Godescalc vers 951 y est recommandé, évoque un projet iconographique pour la façade occidentale de Saint-Jacques de Compostelle incluant une représentation de la Transfiguration ${ }^{16}$. Willibald Sauerländer estime que cette représentation mettait en avant le rôle joué par saint Jacques dans la révélation christique et faisait écho à la disposition des autels à l'intérieur de SaintJacques $^{17}$. Par ailleurs, l'évocation des saints Gilles et Martin dans les chapelles encadrant au nord et au sud le porche de la cathédrale du Puy, pourrait aller dans le sens d'une mise en valeur des axes de pèlerinage vers Saint-Jacques, avec deux autres étapes importantes que sont Tours et Saint-Gillesdu-Gard. Ici, le thème pourrait donc être une évocation du sanctuaire sur la route duquel est située la cathédrale. Les ensembles peints situés dans les parties occidentales de la cathédrale renseignent plusieurs aspects de l'édifice. L'iconographie développée au Puy met en valeur la figure mariale par un lien constant entre sa représentation comme mère du Fils de l'Homme, actrice de l'Incarnation, et le rôle joué dans l'histoire du Salut par le Christ, Fils de Dieu. Les espaces annexes des chapelles latérales et, de façon moins évidente, les peintures du haut du porche, témoignent autant du lien privilégié avec Rome que de l'inscription dans le parcours d'un des pèlerinages majeurs de la Chrétienté, celui de Compostelle. Les décors peints affirment le statut de cet espace comme un espace d'accueil et de départ, un lieu de passage pour les pèlerins, créant ainsi un dialogue entre l'espace intérieur et le culte rendu à la Vierge et l'espace extérieur, le pèlerinage, cheminement spirituel vers les grands centres de la Chrétienté.

\section{LES PEINTURES DES PARTIES ORIENTALES ET LA LITURGIE CATHÉDRALE}

Les peintures de l'intérieur de la cathédrale se situent principalement dans le transept. Les thèmes abordés semblent directement liés aux célébrations liturgiques dans le chœur et devant divers autels secondaires. Le chantier a d'abord concerné les tribunes nord et sud du transept, puis le bras nord. Les peintures conservées au nord et les relevés et bribes conservées des peintures de la tribune sud ont probablement été peintes en une seule phase de chantier pouvant être située durant la seconde moitié du XI ${ }^{\mathrm{e}}$ siècle. Dans la tribune nord, les peintures occupent les parois occidentale, septentrionale et orientale. Elles se développent autour des baies ainsi que sur les intrados des arcades. La paroi occidentale est couverte, dans sa partie septentrionale, par un immense saint Michel (fig. 12), disposé au-dessus de la porte et surmonté par deux cerfs affrontés. Sur le mur nord, court un registre où est représenté le Jugement de Salomon, accompagné d'une figure de saint. L'intrados de la fenêtre comporte deux registres occupés par une figure de soldat en bas et de saint en haut. La paroi nord est rythmée par deux arcs aveugles, occupés dans leur partie supérieure par deux baies encadrées de motifs d'arbres. Les registres endessous de ces baies sont consacrés à deux scènes identifiées par Marcel Durliat comme le Martyre de Naboth de Jezraël à gauche et la Mort de Jézabel à droite $\left(\mathbf{f i g}\right.$. 13) ${ }^{18}$. La paroi orientale est, pour sa part, divisée en quatre zones. La première consiste en une arcade aveugle étroite, portant encore des traces de polychromie : un motif d'entrelacs répété au niveau de l'intrados. Deux retombées d'arcs doubleaux viennent encadrer une arcature dans laquelle s'inscrivent une baie et une porte, percée a posteriori (fig. 14). Elles sont recouvertes de trois registres à gauche et de quatre à droite.

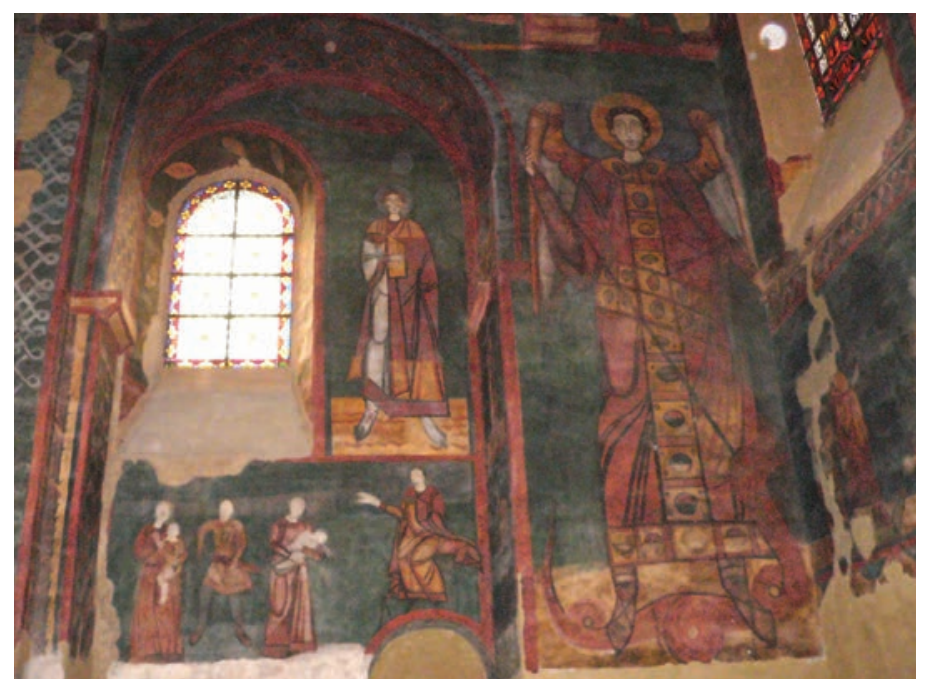

Fig. 12 - Tribune nord, mur ouest (cl. M. Charbonnel)

\footnotetext{
${ }^{15}$ Le manuscrit a été publié par U. CHEVALLIER qui pointait déjà l'antériorité certaine du texte : Prosolarium Ecclesiae Aniciensis : office en vers de la circoncision en usage dans l'église du Puy, Paris, 1894, p. 37 ; W. ARLT, The Office for the Feast of Circumcision from Le Puy, in M. E. FASSLER et R. A. BALTZER (éd.), The Divine Office in the Latin Middle Ages: Methodology and Source Studies, Regional Developments, Hagiography, Oxford, 200o, p. 324-343. ${ }^{16}$ C. LAURANSON-ROSAZ, Gotiscalc, évêque du Puy (928-962), in Retour aux sources. Textes, études et documents d'histoire du Moyen Âge offerts à Michel Parisse, Paris, 2004, p. 653-667.

${ }_{17}$ W. SAUERLÄNDER, Reliquien, Altäre und Portale, in Kunst und Liturgie im Mittelalter. Akten des internationalen Kongresses der Bibliotheca Hertziana und des Nederlands Instituut te Rome (Rom, 28.-30. September 1997), Rome, 2000, p. 122 ; J. VIEILLARD (éd.), Le guide du pèlerin de Saint-Jacques de Compostelle. Texte latin du XII e siècle, édité et traduit en français d'après les manuscrits de Compostelle et de Ripoll, $5^{\mathrm{e} e ́ d i t i o n, ~ P a r i s, ~ 2004, ~ p . ~ 102-105 . ~}$

${ }^{18}$ M. DURLIAT, op. cit. (n. 1), p. 115-116.
} 




Fig. 13 - Tribune nord, mur nord (cl. M. Charbonnel)

Ceux-ci sont consacrés à des évêques et des martyrs malheureusement impossibles à identifier en raison de la perte des inscriptions qui les accompagnaient. Concernant l'arcade, seule la partie haute et l'intrados possèdent des traces de polychromie. Ainsi, deux personnages flanquent la baie ('un d'eux porte l'inscription Sanctus [...]nestus) ${ }^{19}$, tandis que cinq autres - trois à gauche et deux à droite - tenant des phylactères, occupent l'intrados, et viennent encadrer une main bénissant.

Plus rien ne subsiste aujourd'hui des peintures de la tribune méridionale, excepté leur description par Aymond Mallay et les relevés d'Émile Giraud exécutés en $1844^{20}$. D’après ces documents, la tribune sud a également comporté des figures de prophètes : Moïse, David, Samuel, Zacharie, Sophonie et Michée, ainsi qu'une Crucifixion sur le mur oriental. Les prophètes sont tous identifiés et accompagnés de citations, relevées de manière fautive par Émile Giraud mais néanmoins identifiables. Ainsi, chaque prophète tient un phylactère sur lesquels sont inscrites les citations suivantes :

Moïse : Prophetam de gente tua et de fratribus tuis sicut me suscitabit tibi Dominus²1 (Deut. XVIII, 15).

David : Induti sunt arietes ovium et valles abundabunt ${ }^{22}$ (Ps LXIV, 14).

Zacharie: Exulta filia Sion. Ecce rex tuus veniet tibi iustus et salvator ipse pauper, ascendus super asinum pullum filium asinae ${ }^{23}$ (Za IX, 9).

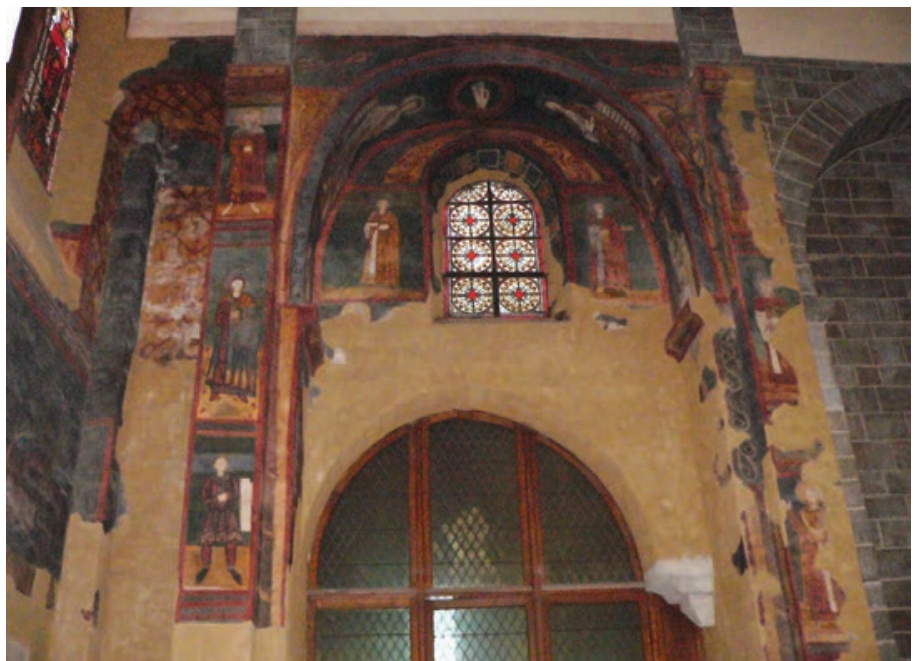

Fig. 14 - Tribune nord, mur est (cl. M. Charbonnel)

Sophonie : Lauda filia Sion. Iubilate Israel laetare et exulta in omni corde filia Hierusalem ${ }^{24}$ (So IV, 14).

Michée : Principium peccati est filie Sion quia inte inventa sunt scelera Israel25 (Mi I, 13).

Habacuc était également représenté, mais l'inscription de son phylactère ne nous est pas parvenue. Le rôle des prophètes est renforcé par leur association à quelques scènes $\mathrm{du}$ Nouveau Testament : l'Entrée du Christ à Jérusalem à l'ouest, accompagnée du commentaire Occurrunt pueri cantant [---] vemiu [---] Deum contempnit crede[..] rever[--- et la Cène au sud ${ }^{26}$. Ces dernières font elles-mêmes pendant à des scènes de l'Ancien Testament, Salomon accueillant la reine de Saba à l'ouest et Moïse faisant jaillir l'eau du rocher au sud. Une Crucifixion ferait quant à elle écho à la figure de Moïse face au serpent d'airain. Enfin, une peinture faisait la synthèse des différentes scènes, avec une représentation de l'Ancienne et de la Nouvelle Loi, à savoir Moïse recevant les tables de la Loi sur lesquelles est inscrite la citation Diliges Dominum Deum tuum ${ }^{27}$ et Jésus enseignant aux apôtres, identifiés par l'inscription Apostoli Dominis et accompagnés de la citation de Jean XX, 22, Accipite spiritum sanctum ${ }^{28}$. Un commentaire est inscrit en dessous de la scène :--- $] a[---]$ cus simulacrae pecatur. $\{D(\mathrm{omi}) n(u)$ s legem priscam $\}$ complet nunc ipse [--- $]^{29}$.

La mise en concordance entre Ancien et Nouveau Testaments est ici clairement affichée, tant dans les images que dans les inscriptions. Néanmoins, il convient, afin de cerner

\footnotetext{
${ }_{19} S($ an $) c($ tu $)$ s [...]nestus.

${ }^{20}$ V. GOUTAYER, L'iconographie du groupe cathédral du Puy, Mémoire de D.E.A. sous la direction de J.-P. CAILLET, Université de Paris X, 2001 ; les relevés de toutes ces peintures sont reproduits dans le catalogue Regard du XIXe siècle sur les fresques de la cathédrale du Puy, Le Puy-en-Velay, 2005, p. 110-121.

${ }^{21}$ Prophe(t)am de gente tua et de fratribus tuis sicut me su\{s\}citabit tibi $D($ omi $) n(u) s$.

${ }^{22}$ Induti su\{n\}t ar(ie)tes ovi $\{$ um\} $\}$ et va $\{l l\}$ es abu(n)da\{bunt\}.

${ }^{23}$ Exul $\{t a\}$ fil $\{i\} a$ Sion. Ecce rex $\{$ tuus veniet $\}$ tib(i) iustus et sa(l)vat $\{$ or $\}$ i $\{$ pse pauper $\}$, ascen $\{$ dus super asinum\} pullum filium asinae.

${ }^{24}$ Lauda fil $\{i\} a$ Sion. Iubilat $\{e\}$ Israe $\{l$ lae $\}$ tare et exulta in $\{0\} m n\{i$ c $\}$ or $\{d\}$ filia Hi $\{e\} r u s a\{l\} e m$.

${ }_{25}$ Principiu(m) peccati est filie Sion [quia inte inventa sunt scelera Israel].

${ }^{26}$ Il convient toutefois de se prémunir d'une possible erreur de lecture - elles sont nombreuses pour les citations des prophètes - du copiste Émile Giraud. Aymond Mallay, dans sa description, donne le même formulaire, mais il peut se fonder sur la lecture du copiste.

${ }_{27}$ Dilige(s) Domin(u)m De(um) [t]uum.

${ }^{28}$ A $\{$ c $\}$ cipite $[$ s]piritum $s($ an $) c(t u) m$.

${ }^{29}$ L'ordre des mots a été modifié par rapport au relevé d'Émile Giraud qui donne ---]A[---]CUSSIMUIACRAE PECATVR . LEGEM DANS PRISCAM COMPLET NVNC IPSE CIS.
} 
pleinement les enjeux liés à ces tribunes de transept, de lier les deux ensembles. En effet, la tribune nord, au-delà de la probable titulature à saint Michel et du caractère protecteur de ce dernier, semble axée sur les figures hagiographiques. L'ensemble forme une sorte de litanie plastique. La tribune sud semble, pour sa part, clairement tournée vers la figure christique. Cette bipartition pourrait être mise en relation avec l'utilisation des tribunes pour le chant liturgique, avec un jeu de réponses entre Kyrie et Christe eleison, dans la messe et lors des célébrations du Jeudi saint. Le Kyrie eleison, considéré comme la prière universelle, est introduit dans la messe entre l'introït et le Gloria dès le concile de Vaison, tenu en 529, et associé aux litanies ${ }^{30}$. L'alternance entre Kyrie et Christe eleison est, pour sa part, mentionnée dans le Quotiens contra se au IX ${ }^{\mathrm{e}}$ siècle pour la messe. Cette pratique est bien documentée dans la Regularis Concordia pour la période pascale :

Evangeliique antiphona finita nihilque iam cereorum luminis remanente, sint duo ad hoc idem destinati pueri in dextera parte chori qui sonora psallant voce Kyrie Eleison, duoque similiter in sinistra parte qui respondeant Christe Eleison, nec non et in occidentali parte duo dicant Domini miserere nobis ; quibus peractis respondeat simul omnis chorus ${ }^{31}$.

La formule épigraphique qui est développée sous l'Entrée du Christ à Jérusalem doit d'ailleurs nous interroger sur une utilisation des tribunes par les chœurs d'enfants. En effet, le pueri ... clamabant des antiennes du dimanche des Rameaux s'est ici transformé en occurunt pueri cantant ${ }^{32}$. Si l'on associe une telle variation lexicale à une particularité du dispositif liturgique, l'emploi du verbe cantare prend une toute autre dimension et devient une allusion à la forme d'expression vocale du rituel ici employée. Le mot pueri devient une allusion aux acteurs de cette manifestation. D’autre part, le clamabant est associé dans une antienne du dimanche des Rameaux à la citation de Zacharie donnée plus haut :

Cum audisset populus quia Jesus venit Jerusalem acceperunt ramos palmarum et exierunt ei obviam et clamabant pueri dicentes hic est qui venturus est in salutem populi haec est salus nostra et redemptio Israel quantus est iste cui throni et dominationes occurrunt noli timere filia Sion ecce rex tuus venit tibi sedens super pullum asinae sicut scriptum est salve rex fabricator mundi qui venisti redimere nos ${ }^{33}$.
Le prophète est d'ailleurs proche de l'Entrée du Christ. Les pueri chori constituent une composante essentielle de la liturgie chantée en général, et jouent un rôle central dans la liturgie du Dimanche des Rameaux ${ }^{34}$. Ainsi, à la cathédrale Notre-Dame de Reims, au XII e siècle, lors de la procession du Dimanche des Rameaux, les enfants précèdent les chanoines dans l'église et montent dans les tribunes occidentales (absentes au Puy) pour entonner le Gloria laus, qui est ensuite repris par les chanoines dans l'atrium ${ }^{35}$. Leur rôle pourrait donc avoir été valorisé dans les inscriptions et l'iconographie de la paroi occidentale de la tribune du Puy. Les inscriptions mettent de la voix dans l'image à travers des paroles, comme celles des prophètes, et dans l'évocation de la performance chantée, à travers le commentaire de l'Entrée du Christ à Jérusalem. Ces développements picturaux permettent également de prendre la mesure des deux temps de réactivation de la mémoire pascale, entre célébrations annuelles et quotidiennes au sein de l'édifice.

Enfin, dans le cadre de ces peintures, retenons l'importance de leur date présumée, proposée par Marcel Durliat et reprise ici, proche de la première Croisade. Les armées étaient alors dirigées par Adhémar de Monteil, évêque du Puy et légat du pape Urbain II $^{36}$. L'étude d'Anne Derbes tend à démontrer qu'un lien peut être établi entre la personnalité d'Adhémar de Monteil et ces peintures, notamment dans la comparaison faite par certains auteurs médiévaux tels que Raoul de Caen et Raymond d'Aguilers, entre le légat et la figure de Moïse, très présente dans cette tribune ${ }^{37}$. Néanmoins, sans rejeter cette interprétation, force est de constater qu'il n'est pas rare d'associer Moïse à des transcriptions visuelles des célébrations de la Semaine Sainte. Des éléments comme la croix en ivoire conservée au Cloisters Museum de New York sont là pour en témoigner ${ }^{3^{8}}$.

Des peintures, datables du début du XIII siècle, occupent également deux absidioles conjointes à l'extrémité septentrionale du bras nord du transept, ainsi que le mur sud de la salle attenante au cloître. La liturgie et la commémoration de la période pascale sont également au cœur de l'iconographie et de l'épigraphie des peintures de l'absidiole occidentale, occupée par une représentation des saintes Femmes au tombeau (fig. 15). Les trois femmes arrivent au tombeau que leur indique l'ange. Une inscription accompagne la scène, sous le linceul vide désigné par l’ange : Surrexit, non est hic. Il s'agit d'un trope du drame liturgique de la Visitatio Sepulcri et plus particulièrement la séquence du Quem quaeritis. Le

\footnotetext{
30 G. IVERSEN, Chanter avec les anges : poésie dans la messe médiévale, interprétations et commentaires, Paris, 2001, p. 75-104 ; E. H. KANTOROWICZ, Laudes Regiae : une étude des acclamations liturgiques et du culte du souverain au moyen âge, Los Angeles, 1946, trad. Alain Wijffels, Paris, 2004.

${ }^{31}$ B. FRANZÉ, La pierre et l'image. L'église de Saint-Chef-en-Dauphiné, Paris, 2010, p. 110, d'après The monastic agreement of the Monks and the Nuns of the English Nation, trad. du latin en anglais avec introduction et notes de T. SYMONS, T. NELSON, Londres-Édimbourg, 1953, p. 36.

${ }^{32}$ La formule la plus proche est celle de l'antienne de la Pentecôte (C.A.O. 1473) : Ante sex dies sollemnis paschae quando venit dominus in civitatem Jerusalem occurrerunt ei pueri et in manibus portabant ramos palmarum et clamabant voce magna dicentes hosanna in excelsis benedictus qui venisti in multitudine misericordiae hosanna in excelsis benedictus qui venisti in multitudine misericordiae hosanna in excelsis.

33 C.A.O. 1983.

${ }^{34}$ P. DEMOUY, Les pueri chori de Notre-Dame de Reims. Contribution à l'histoire des clergeons au Moyen Âge, in Clerc séculier au Moyen Âge, Actes du $22^{e}$ congrès de la Société des historiens médiévistes de l'enseignement supérieur public (Amiens), 1991, p. 136-149.

35 Ibid., p. 138.

${ }^{36}$ M. DURLIAT, op. cit. (n. 1).

37 A. DERBES, A Crusading Fresco Cycle at the Cathedral of Le Puy, in The Art Bulletin, 73, nº 4, déc. 1991, p. 561-576. L'auteure cite ces vers de Raoul de Caen : Conditus est Moysis clarissimus hic imitator/ Doctrina, studio, moribus, officio/ Dux populi Moyses, et dux populi fuit iste/ Ambo duces Christi, coelitus ambo sati/ Ambo justitiae, doctrinae ambo studiosi/ Ambo fuere Dei vox media et populi.... d'après le Recueil des Historiens des Croisades. Historiens occidentaux, Paris, 1844-1895, III, 673-74.
}

$3^{8}$ E. C. PARKER, C. T. LITTLE, The Cloisters Cross. Its art and meaning, New York, 1994, p. 149-174. 
voile, bien valorisé, est d'ailleurs désigné ici par l'ange, comme dans le drame liturgique $^{39}$. Or, ce drame est étroitement lié aux espaces de tribunes, commeà Fleury ou encore dans la Regularis Concordia d'Etthelword $^{40}$. Le déroulement de la séquence débute pendant la récitation de la troisième leçon du Vendredi saint. Quatre frères vont alors s'habiller, le premier met une aube blanche et s'assoit au lieu du sépulcre, les trois autres portent de l'encens et s'approchent plus tard du premier $^{41}$. Il est précisé que tout ceci se fait à l'imitation de l'ange et des saintes Femmes ${ }^{42}$. Un échange de paroles s'opère alors comme suit, dans lequel se trouve la citation développée sur la peinture de l'absidiole :

Quem quaeritis (in sepulchro, $O$ Christicolae)?

Jhesum Nazarenum (crucifixum, o coelicola).

Non est hic, surrexit sicut praedixerat, ite, nuntiate quia surrexit a mortuis.

Alleluia, resurrexit Dominus, hodie resurrexit leo fortia Christus, filius Dei.

Soulevant ensuite un rideau, l'ange dit : Venite et videte locum ubi positus erat Dominus, alleluia. Les saintes Femmes prennent alors le linceul, mis en valeur dans la peinture murale du Puy, et l'étendent vers le clergé avant de le déposer sur l'autel en chantant Surrexit Dominus de Sepulchro, qui pro nobis pependit in ligne, alleluia. L'hymne Te deum laudamus est ensuite chanté puis les cloches sont actionnées.

Cette peinture témoigne d'un dispositif liturgique fréquemment associé à l'espace du transept et à la séquence liturgique du Quem quaeritis. Ce lien a laissé d'autres traces dans la pierre dans des lieux aussi divers que Saint-Just de Ségovie ou la basilique Santa Maria Assunta d'Aquilée. Il faut noter qu'à Saint-Just de Ségovie, l'évocation du SaintSépulcre prend la forme d'une tour accolée au bras nord du transept qui conserve le Cristos de los Gascones, Christ en croix articulé du milieu du XII ${ }^{e}$ siècle, probablement utilisé

lors des célébrations liturgiques de la Semaine Sainte où le Christ était allongé dans le sépulcre, les bras repliés ${ }^{43}$. De plus, l'entrée de la tour est dotée d'un tympan, témoignant d'une monumentalisation de l'intérieur, a fortiori de l'entrée d'une chapelle annexe, peu courante. Ce tympan est le support d'une représentation des Saintes Femmes au tombeau, derrière laquelle se trouve la figuration d'un évêque trônant, probablement le commanditaire de l'œuvre. Certains auteurs dont Eduardo Carrero Santamaría proposent de voir dans cette scène une représentation de l'Invention de la Vraie Croix. Quelques détails iconographiques paraissent néanmoins troublants, comme la présence de l'ange portant l'encensoir, les pots à onguents des suivantes et le fait que l'évêque ne paraisse pas réellement intégré à la scène. La figuration avec la Croix au-dessus du tombeau et l'encensoir est d'ailleurs proche de la représentation des saintes Femmes du bas-relief de Saint-Paul de Dax. D'autres exemples de Saint-Sépulcre, tels ceux d'Aquilée ou de Gernrode, peuvent allonger la liste de tels dispositifs au nord des édifices ${ }^{44}$.

Au Puy, l'espace du transept serait ainsi profondément lié au temps des célébrations du Triduum sacrum. Par son

39 E. CARRERO SANTAMARÍA, El Santo Sepulcro. Imagen y funcionalidad espacial en la capilla de la iglesia de San Justo (Segovia), in Annuario de estudios medievales, 27-1, 1997, p. 461-478 ; J. I. GONZÁLEZ-MONTAÑÉS, Drama e iconografía en el arte medieval peninsular (siglos XI-XV), Thèse de doctorat sous la direction de V. N. ALCAIDE, UNED - Madrid, 2002.

${ }^{40}$ E. C. PARKER, Architecture as liturgical setting, in T. J. HEFFERNAN, E. A. MATTER, The liturgy of the medieval Church, Kalamazoo, 2001, p. 292 ; B. FRANZÉ, op. cit. (n. 30), p. 110. Pour l'association au pôle occidental des églises-porches voir C. HEITZ, Architecture et liturgie : le saint Sépulcre en Occident, in Les Cahiers de Saint-Michel de Cuxa, 22, 1991, p. 43-56. Sur la pérénité de ce schéma liturgique voir H. HUBACH, “... scrinium super sepulchrum aperiuntur": Die Heilig-Grab-Kapelle der Aschaffenburger Stiftskirche und Matthias Grünewalds "Beweinung Christi", in "Ich armer sundiger Mensch". Heiligen- und Reliquienkult am Übergang zum konfessionellen Zeitalter, Göttingen, 2006, p. 415-498.

${ }^{41}$ Dum tertia recitatur lectio, iiii fratres induant se, quorum unus alba indutus acsi ad aliud agendum ingrediatur, atque latenter sepulcri locum adeat, ibique, manu tenens palmam, quietus sedeat. Dumque tertium percelebratur responsorium, residui tres succedant omnes quidem cappis induti, turribula cum incensu manibus gestantes, ac pedetemptim ad similitudinem quaerentium quid, veniant ante locum Sepulcri. D'après K. YOUNG, The Drama of the Medieval Church, Oxford, 1933, I, p. 183.

${ }^{42}$ Aguntur enim haec ad imitationem angeli Sedentis in Monumento atque mulierum cum aromatibus venientium ut ungerent corpus Jhesu. Cum ergo ille residens tres velut erraneos ac aliquid quaerentes viderit sibi adproximare, incipiat mediocri voce dulcisone cantare: Quem quaeritis (in sepulchro, $O$ Christicolae)? Quo decantato fine tenus, respondeant hi tres uno ore :Jhesum Nazarenum (crucifixum, o coelicola). Quibus ille: Non est hic, surrexit sicut praedixerat, ite, nuntiate quia surrexit a mortuis (ibidem, loc. cit.).

43 E. CARRERO SANTAMARÍA, op. cit. (n. 38), p. 463.

${ }^{44}$ E. DYGGVE, Aquileia e la Pasqua, in Studi aquileiesi offerti a G. Brusin, Aquilée, 1953, p. 385-397 ; P. L. ZOVATTO, Il Santo Sepolcro di Aquileia e il dramma liturgico medievale, in Atti dell'Academia di Udine, IV-13, 1954-1957, p. 127-151 ; S. PIUSSI, Il Santo Sepolcro di Aquileia, in Aquileia e l'Oriente mediterraneo, Antichità altoadriatiche, 12, Udine 1977, II, p. 511-559. 


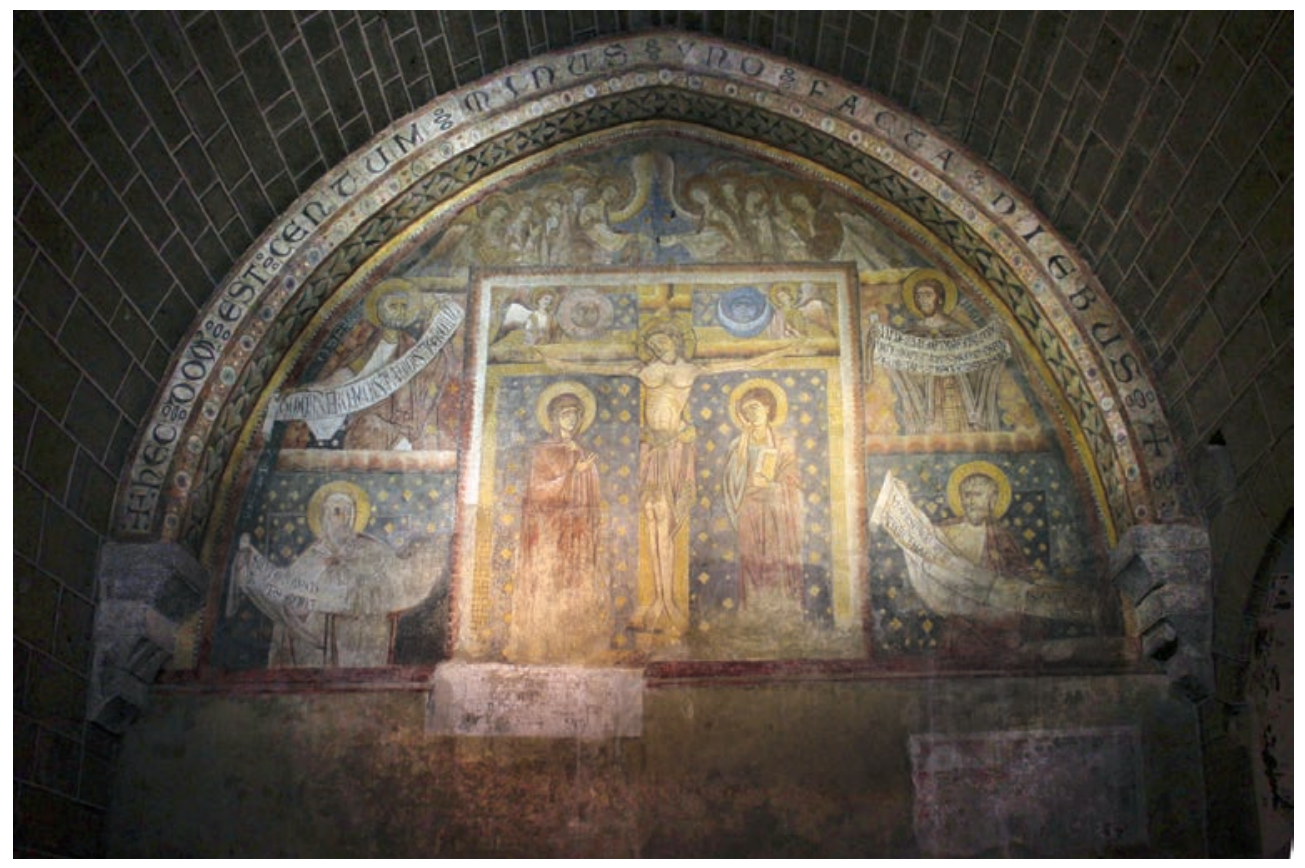

Fig. 16 - Cloître, salle dite des morts, la Crucifixion (cl. M. Charbonnel)

Au-dessus d'eux se trouvent deux anges avec le soleil et la lune tandis que dix anges, répartis à raison de cinq par côté se partagent le sommet. Les éléments les plus rares se situent de part et d'autre du groupe central ainsi que sur l'archivolte qui chapote l'ensemble de la composition. Aussi, quatre personnages se partagent les parties latérales. À gauche au niveau du registre supérieur, se trouve le prophète Osée ${ }^{45}$ qui tient un phylactère sur lequel est inscrite la citation suivante (Osée, XIII, 14) : O mors ero mors tua mors(us) tu(us) ero inferne. En dessous de lui se tient Isaïe ${ }^{46}$ qui porte lui aussi un phylactère avec l'inscription (Isaïe, LIII, 7) : Sicut ovis ad occis[ionem ducetur et quasi Agnus coram] tondente se obmutuit et [non aperuit os suum]. En haut à droite, Philon ${ }^{47}$ s'exprime lui aussi à

interaction avec les gestes et les paroles des personnages du drame liturgique de la Visitatio sepulcri, l'image de l'absidiole fait écho au rituel annuel dont elle renforce le sens en accentuant son inscription dans l'espace et dans le temps. Les peintures participent d'une recréation du lieu saint qu'est Jérusalem, démarche d'autant plus répandue dans cette période de croisade. Elles participent également, par leur rôle paraliturgique, de la réactivation d'un temps, de la memoria Christi, de sa Résurrection par ses contenus iconographique et épigraphique. Ainsi, cette réactivation de la memoria Christi ne se limite pas à une temporalité ponctuelle, celle du Triduum sacrum ; les peintures permettent aussi, par leur caractère permanent et leur exposition dans l'édifice, une réactivation régulière tout au long de l'année. Le support peint présente dans une telle configuration un intérêt majeur puisqu'il réactive potentiellement chez le spectateur le souvenir des célébrations pascales et leur iconographie "en mouvement ». Cette logique verra son aboutissement dans les Mises au Tombeau monumentales de la fin du Moyen Âge où la tridimensionnalité, l'échelle de représentation et une polychromie appuyée serviront cette mémoire régulière et plus douloureuse que glorieuse. Les images et les inscriptions fixent ainsi dans une forme visuelle l'instant liturgique tout en le faisant résonner à l'échelle du temps chrétien.

Il en va de même pour la peinture qui se trouve sur le mur sud de la salle dite des morts, qui servait de lieu de sépulture et probablement de salle capitulaire, dans le prolongement nord du transept. Le thème de cette peinture est une Crucifixion et sa composition est peu courante (fig. 16). Ainsi, la Crucifixion occupe naturellement le centre de la composition, Marie, Jean et le Christ s'inscrivent dans un rectangle. travers son phylactère (Livre de la Sagesse, II, 16) : Dixer(unt) impii opprimam(us) virum j(us)tum inj(us)te morte turpissima condemnem(us) eum tanq(uam) nugaces estimati sum(us) ab illo. Le dernier personnage, en bas à droite, est Jérémie. Son phylactère contient l'inscription lacunaire (Jérémie, XI, 19) : Ego q(ua)si agn(us) m[ansuetus qui portatur] ad victimam [---] Deus meus [---]. Enfin, l'archivolte porte, quant à elle, une mention de construction : Hec dom(us) est ceuntum minus uno facta diebus. Robert Favreau a rattaché les inscriptions de cette peinture aux laudes et matines de la Semaine Sainte ${ }^{4}$. Ainsi, les citations d'Osée et Philon sont des antiennes des laudes du samedi saint (CAO 4045) et du mardi saint (CAO 2263). Celles d'Isaïe et de Jérémie sont des répons des matines du samedi (CAO 7761$)$ et du mardi (CAO 6660) saints. Une nouvelle fois, Pâques, temps majeur de l'année liturgique, laisse une empreinte dans le décor monumental de la cathédrale. Il est également nécessaire de rappeler quà la période d'exécution des peintures, un passage existait entre la quatrième travée du bas-côté nord et cette salle. Ceci laisse envisager une ambulation liturgique entre les deux zones, par ailleurs attestée pour l'office de la Circoncision célébré au nouvel an et connu par une copie de 1553, lors des célébrations de la Semaine Sainte ${ }^{49}$.

Enfin, la seconde peinture du transept nord, à l'est, est consacrée à l'iconographie de sainte Catherine (fig. 17). Un groupe de personnages est représenté à gauche de la peinture, avec, pour l'un d'entre eux, l'inscription Cesar imperator. Ils regardent Catherine durant le supplice de la roue, tandis que l'ange retire les dents de la sainte. Sur l'intrados de l'arc d'entrée de l'absidiole est peint un personnage identifié comme Salomon. Ces peintures accompagnaient probablement un autel dédié aux vierges. Ce 
type de dispositif est souvent présent dans le déambulatoire, comme à la collégiale Notre-Dame d'Aigueperse, avec une iconographie de sainte Catherine, ou à la cathédrale de Clermont, pour la même période, mais aussi à Saint-Savin-sur-Gartempe antérieurement, avec sainte Agathe $^{50}$. En l'absence de chapelles rayonnantes, il est ici déplacé dans le transept. Notons par ailleurs qu'une peinture représentant Dioclétien assistant au massacre des Chrétiens était présente au niveau de la chapelle dite du Crucifix, à l'extrémité orientale du bras nord du transept. Il pourrait s'agir de l'évocation d'une autre catégorie de saints, les martyrs, selon un schéma également présent, entre autres, à Saint-Savin ${ }^{51}$.



Fig. 17 - Transept nord, le Martyre de sainte Catherine (cl. M. Charbonnel)

\section{CONCLUSION}

l'importance du culte, tout en répondant aux attentes des visiteurs.

D’autre part, les peintures témoignent de l'action mnémonique et paraliturgique du support peint, qui se situe au carrefour entre le lieu matériel et les gestes et paroles de l'acte rituel. Elles renforcent ceux-ci par des allusions plus ou moins directes, offrant leur source mémorielle, rappelant leur raison d'être aux yeux des desservants. Elles créent ainsi une componction qui renforce l'efficacité émotionnelle et sensorielle du rite tant chez l'émetteur que chez le récepteur. Deux temporalités - l'une terrestre, liturgique, présente, l'autre céleste, à l'échelle du temps chrétien, entre passé et futur - se rencontrent continuellement dans l'espace religieux, initiant par là même une connexion entre les agents du rituel. Celle-ci est favorisée par les supports plastiques qui permettent d'étendre la portée mnémonique de l'acte rituel dans le temps et d'amplifier sa perception, par leur caractère pérenne et statique. Les relations entre les images, la performance rituelle et sa réception sont ici palpables dans le choix des sujets iconographiques et épigraphiques et dans leur association à des espaces précis. Ces éléments inscrivent l'espace cathédral du Puy dans un hic et nunc liturgique, dans l'instant passé commémoré et dans l'instant à venir souhaité ou redouté.

\footnotetext{
${ }_{50}$ R. FAVREAU, op. cit. (n. 47), p. 21-73.

${ }^{51}$ Ibidem.
} 